\title{
Spherical Wave Positioning based on Curvature of Arrival by an Antenna Array
}

\author{
Siwei Zhang, Member, IEEE, Thomas Jost, Member, IEEE, Robert Pöhlmann, Member, IEEE, \\ Armin Dammann, Member, IEEE, Dmitriy Shutin, Member, IEEE, and Peter Adam Hoeher, Fellow, IEEE
}

\begin{abstract}
Array processing is a key technology for emerging mobile networks, especially in short to moderate range and LOS scenarios. In these scenarios, the incoming wavefront can be modeled by a spherical wave. The wavefront curvature, i.e. Curvature of Arrival (CoA), contains position information of the transmitter and is observable by an antenna array potentially asynchronous and non-coherent to the transmitter. We derive a simplified expression of the spherical wave positioning (SWP) Cramér-Rao bound (CRB) for arbitrary centro-symmetric arrays (CSAs), which provides a geometrical inference about the achievable performance. Additionally, a low complexity CoA positioning algorithm is proposed. In contrast to conventional methods, the proposed algorithm requires neither multiple anchors nor coordination between devices. It also outperforms the Fresnel approximation based SWP algorithms by overcoming the model mismatch. Therefore, the proposed $\mathrm{CoA}$ positioning algorithm is promising for precise positioning in future mobile networks.
\end{abstract}

\section{INTRODUCTION}

Ubiquitous realtime position information is envisaged as a key feature of future mobile networks, for example the $5^{\text {th }}$ generation (5G) networks, due to emerging device-centric applications [1]. Studies have been conducted on $5 \mathrm{G}$ positioning, where $5 \mathrm{G}$ networks provide opportunities for precise positioning in global navigation satellite system (GNSS)-impaired environments [2]. Most traditional positioning techniques exploit either time of arrival (ToA) or direction of arrival (DoA) estimates and demand multiple anchors [3]. Recent research has focused on positioning with a single anchor. The simultaneous localization and mapping (SLAM) algorithm in [4] utilizes multipath components for positioning. However, it requires memory-intensive storage and a static environment. In combined DoA and ToA estimation, an antenna array is used to position a transmitter. With the far-field assumption, distance information is obtained solely from the propagation delay of the baseband pilot signal, whereas the DoA is estimated from the carrier phase differences between antennas [5], [6]. For ToA estimation, the pilot signal structure must be known to the receiver. Additionally, synchronization between the transmitter and the receiver must be ensured.

Large antenna arrays are widely foreseen for 5G applications, varying from dozens of antennas at mobile stations [5] to over one hundred at local access points [7] and up to a few thousands at base stations [1]. These arrays provides

S. Zhang, T. Jost, R. Pöhlmann A. Dammann and D. Shutin are with the Institute of Communications and Navigation, German Aerospace Center (DLR), 82234 Wessling, Germany (e-mail: firstname.lastname@dlr.de).

P. A. Hoeher is with the Chair of Information and Coding Theory, University of Kiel, 24143 Kiel, Germany (e-mail: ph@tf.uni-kiel.de).

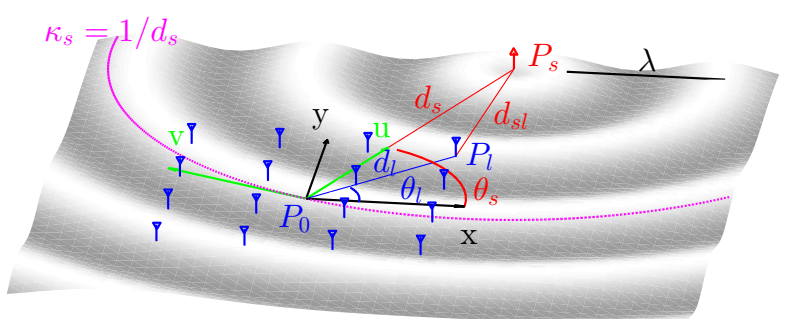

Fig. 1: Spherical wave positioning based on CoA.

communication coverage, from their radiating near field (a few meters) to the beginning of the Fraunhofer region (up to a hundred meters), to mobile devices under line-of-sight (LoS) condition. The signal wavefront received by the array is modeled by a spherical wave. Under this model, not only DoA but also distance information of the transmitter is contained in the carrier phase, which enables SWP of the transmitter [7]-[10]. Most previous works apply the Fresnel approximation to arrays with special geometries, e.g. uniform linear arrays (ULAs) [8]-[10], and introduce a model mismatch. This mismatch has recently been noticed to jeopardize the achievable positioning precision [11]. In [12] a lookup table is used for ULA model correction. The maximum likelihood (ML) algorithm in [7] exploits the exact model, but includes a computationally expansive recursion. In [13] the CRB of SWP is analyzed for special array geometries.

In this work, a simplified expression of the CRB of SWP is derived for arbitrary CSAs, which only depends on the relative geometry and characteristics of the antennas' spatial distribution. This expression provides a geometric inference about the achievable performance and brings further insights into array design. Additionally, we propose a method dubbed CoA positioning, which extracts the transmitter position information directly from the wavefront curvature. CoA positioning overcomes the model mismatch introduced by Fresnel approximation, while maintaining low complexity for realtime.

\section{Position Information in Spherical WaVe}

A single transmitter antenna is placed at point $P_{s}$, which radiates a single-carrier signal at carrier frequency $f_{\mathrm{c}}$ with an unknown real-valued amplitude $S$ and an unknown phase $\phi_{\delta}$. The signal propagates under LoS condition to a generic point $P$ at distance $d$ with speed of light $c_{0}$. The signal phase at $P, \phi=\phi_{\delta}-\omega_{\mathrm{c}} d / c_{0}$, is a continuous function in space, where $\omega_{c}=2 \pi f_{c}$. The transmitter's position information w.r.t. an observation point $P_{o}$ can be extracted 
from the continuous wavefield. We define a two-dimensional (2D) Cartesian coordinate system ${ }^{1} \mathcal{C}^{(x y)}$ that originates at point $P_{o}$ and includes point $P_{s}$. The corresponding polar coordinate system is defined as $\mathcal{C}^{(d \theta)}$. We consider the $2 \mathrm{D}$ positioning problem, i.e. estimating $\mathbf{p}_{s}^{(d \theta)}=\left[d_{s}, \theta_{s}\right]^{T}$, where $d_{s}$ is the distance between points $P_{s}$ and $P_{o}$, and $\theta_{s}$ is the DoA w.r.t. the positive $x$-axis of $\mathcal{C}^{(x y)}$. For the rest of the paper, we use $\mathcal{C}^{(d \theta)}$ as the default coordinate system and omit the superscript $(d \theta)$ for simplicity. The transmission phase $\phi_{\delta}$ and signal amplitude $\alpha_{s}$ after propagation are not of interest, but need to be estimated jointly with $\mathbf{p}_{s}$. The total parameter vector to be estimated is $\chi=\left[\mathbf{p}_{s}^{T}, \phi_{\delta}, \alpha_{s}\right]^{T}$. The spherical wave intersects the $x y$-plane with co-phase circles centered at $P_{s}$. The curvature of these circles contains information about the distance to the transmitter. We apply a coordinate transformation $\mathcal{C}^{(x y)} \stackrel{P_{o}, \theta_{s}}{\longmapsto} \mathcal{C}^{(u v)}$, where the new Cartesian coordinate system $\mathcal{C}^{(u v)}$ originates at $P_{o}$ and the $u$-axis is aligned with $\theta_{s}$.

Definition 1 (Signal CoA). The signal CoA $\kappa_{o}$ at point $P_{o}$ is defined as the extrinsic curvature of $-\phi c_{0} / \omega_{c}$ along the $v$-axis of $\mathcal{C}^{(u v)}$. With the spherical wave model, CoA is propotional to the absolute value of the phase's second-order derivative and equals to the reciprocal of $d_{s}$

$$
\kappa_{o} \triangleq-\left.\frac{c_{0}}{\omega_{c}} \frac{\partial^{2} \phi}{\partial v^{2}}\right|_{P_{o}}=\frac{1}{d_{s}} .
$$

The distance information can be extracted from the secondorder derivative of the signal phase. The DoA needs to be estimated prior to coordinate transformation. In practice, a $2 \mathrm{D}$ antenna array on the $x y$-plane, potentially asynchronous and non-coherent to the transmitter, with $L$ elements centered at $P_{o}$ is used to sample the continuous wavefield at discrete spatial points. An element $l, l=1, \ldots, L$, located at point $P_{l}$, receives the baseband signal ${ }^{2}$

$$
r_{l}(t)=\alpha_{s} e^{j \phi_{\delta}} e^{-j \omega_{c} d_{s l} / c_{0}}+n_{l}(t),
$$

where $n_{l}(t) \sim \mathcal{C N}\left(0, \sigma^{2}\right)$ is an i.i.d. circularly-symmetric complex normally distributed noise process. According to the geometry under investigation, $d_{s l}$ can be expressed as

$$
d_{s l}=\sqrt{d_{s}^{2}+d_{l}^{2}-2 d_{s} d_{l} \cos \left(\theta_{l}-\theta_{s}\right)} .
$$

The received samples $r_{l}$ are acquired at an arbitrary time point, coherently at all elements, with a received sample phase $\phi_{l}$. The concept of the SWP with CoA is illustrated in Fig. 1.

\section{FUNDAMENTAL LIMITS OF SWP}

The Fisher information matrix (FIM) of $\chi$ can be calculated from the given model (2) and (3), similarly as in [13]. The covariance of the transmitter position estimate $\operatorname{COV}\left[\hat{\mathbf{p}}_{s}\right]$ is bounded by the $\mathrm{CRB}, \mathrm{CRB}\left[\mathbf{p}_{s}\right]$, which is obtained by applying the Schur complement [6] to the position corresponded

\footnotetext{
${ }^{1}$ The coordinates of a specific point $P_{\epsilon}$ in a coordinate system $\mathcal{C}^{(\xi \psi)}$ are defined as $\mathbf{p}_{\epsilon}^{(\xi \psi)}=\left[\xi_{\epsilon}, \psi_{\epsilon}\right]^{T}$, where $\xi$ and $\psi$ are the two dimensions of that coordinate system. The subscript $\epsilon$ is omitted for generic points $P$.

${ }^{2}$ We assume the array aperture to be much smaller than $d_{s}$. Therefore, the distance-related attenuation differences among elements are negligible.
}

sub-matrix of the FIM. Assuming free-space pathloss, i.e. $\alpha_{s}=S c_{0} / 2 \omega_{c} d_{s}$, the positioning CRB states

$$
\begin{aligned}
& \operatorname{COV}\left[\hat{\mathbf{p}}_{s}\right] \succcurlyeq \operatorname{CRB}\left[\mathbf{p}_{s}\right]=\frac{2 \sigma^{2} d_{s}^{2}}{S^{2}} \\
& \times\left(\left(\sum_{l=1}^{L} \frac{\partial d_{s l}}{\partial \mathbf{p}_{s}} \frac{\partial d_{s l}}{\partial \mathbf{p}_{s}^{T}}\right)-\frac{1}{L} \sum_{l=1}^{L} \frac{\partial d_{s l}}{\partial \mathbf{p}_{s}} \sum_{m=1}^{L} \frac{\partial d_{s m}}{\partial \mathbf{p}_{s}^{T}}\right)^{-1} .
\end{aligned}
$$

To infer the geometry impacts on SWP, we first investigate the symmetric linear array (SLA) case. An SLA is deployed along the $x$-axis, with $L$ elements and an aperture length $A$. We define the $k^{\text {th }}$ moment of the normalized antennas' spatial distribution $M_{k}=\sum_{l=1}^{L}\left(d_{l} / A\right)^{k} / L$, and the effective aperture length $\tilde{A}=A \sin \theta_{s}$, to characterize the array geometry.

Theorem 1 (CRB of SWP for SLA). For the SLA, assuming $L \gg 1$ and $d_{s} \gg A$, the CRB of DoA estimate can be approximated by

$$
\mathrm{CRB}\left[\theta_{s}\right] \approx \frac{2 \sigma^{2} d_{s}^{2}}{S^{2} L \tilde{A}^{2} M_{2}},
$$

whereas the distance CRB is approximated by

$$
\mathrm{CRB}\left[d_{s}\right] \approx \frac{2 \sigma^{2} d_{s}^{2}}{S^{2}} \frac{4 d_{s}^{4}}{L \tilde{A}^{4}\left(M_{4}-M_{2}^{2}\right)} .
$$

Proof. See Appendix.

The array's aperture is often physically constrained by applications. For a fixed $A$, more elements can be deployed for higher carrier frequencies, without resulting in severe antenna mutual coupling. Both CRBs in (5) and (6) linearly decrease with the number of elements $L$, which shows a benefit of higher $f_{c}$, such as foreseen in $5 \mathrm{G}$. The term $2 \sigma^{2} d_{s}^{2} / S^{2}$ shows the effect of the signal-to-noise ratio (SNR). The CRB for DoA decreases quadratically with $\tilde{A}$ and linearly with $M_{2}$, the antennas' spatial spread. The distance CRB experiences a quartic growth with the ratio $d_{s} / \tilde{A}$, indicating a strong impact from the relative geometry. Additionally, it decreases with $M_{4}-M_{2}^{2}$, i.e. the shape of the antennas' spatial distribution. More importantly, when $\theta_{s}=0^{\circ}$, both CRBs approach infinity. Hence the array's aperture expanded in $u$ direction contains no information of the transmitter's position. With the last observation, we extend Theorem 1 to arbitrary 2D CSAs. Many typical arrays are centro-symmetric, e.g. uniform circular/linear arrays, the ones in [13], as well as the uniform rectangular array (URA) illustrated in Fig. 1.

Corollary 1. A CSA centered at $P_{0}$ can be projected on the $v$-axis, forming a virtual SLA. The positioning CRB can be approximated by applying Theorem 1 to the virtual SLA.

Proof. Since the aperture expanded in $u$ direction does not contain position information, the projected virtual linear array along $v$-axis is equivalent to the original CSA in the sense of SWP. By the definition of centro-symmetry, for any noncentered element $l$ with position $\mathbf{p}_{l}=\left[d_{l}, \theta_{l}\right]^{T}$, there exists an element $m$ with position $\mathbf{p}_{m}=\left[d_{l}, \theta_{l}+\pi\right]^{T}$. Elements $l$ and $m$ are projected on the $v$-axis at $\pm d_{l} \sin \left(\theta_{l}-\theta_{s}\right)$ respectively and are symmetric w.r.t. $P_{0}$. Hence the projected array is an SLA, which meets the condition of Theorem 1. 


\section{Low Complexity CoA Positioning Algorithm}

We propose a low complexity CoA positioning algorithm, which avoids recursions and reduces the model error from the Fresnel approximation. It can be applied either directly as a realtime positioning variant or to initialize a recursive algorithm like an ML estimator [7].

We define tiles $\mathcal{T}_{i}$ composed of at least three adjacent antenna elements and centered at points $P_{i}$. The estimated local DoA $\hat{\theta}_{s i}$ can be calculated by traditional far-field DoA estimation methods [14], applying the plane wave model on all applicable antenna pairs $l, m$ in $\mathcal{T}_{i}$

$$
\phi_{l m} \approx-\mathbf{e}_{s i}^{T} \mathbf{p}_{l m} \omega_{c} / c_{0}, \quad \forall d_{l m} \omega_{c} / c_{0}<\pi / 2,
$$

where $\mathbf{p}_{l m}=\mathbf{p}_{l}^{(x y)}-\mathbf{p}_{m}^{(x y)}, \phi_{l m}=\phi_{l}-\phi_{m}$ and $\mathbf{e}_{s i}=$ $\left[\cos \theta_{s i}, \sin \theta_{s i}\right]^{T}$. To estimate the tile's curvature $\kappa_{i}$, a coordinate system $\mathcal{C}^{\left(u_{i} v_{i}\right)}$ local to $\mathcal{T}_{i}$ is defined as $\mathcal{C}^{(x y)} \stackrel{P_{i}, \theta_{s i}}{\longmapsto}$ $\mathcal{C}^{\left(u_{i} v_{i}\right)}$. The second order derivative of phase local to $\mathcal{T}_{i}$ can be approximated by a double difference with three adjacent elements $l, m$ and $n$, which leads to a curvature estimate as

$$
\begin{gathered}
\tilde{\kappa}_{l m n}=2 \frac{\Delta_{l m}-\Delta_{m n}}{v_{l m}+v_{m n}}, \text { where } \Delta_{g h}=\frac{\phi_{g h} c_{0} / \omega_{c}+u_{g h}}{v_{g h}}, \\
{\left[\begin{array}{c}
u_{g h} \\
v_{g h}
\end{array}\right]=\left[\begin{array}{c}
\hat{\mathbf{e}}_{s i}^{T} \\
\hat{\mathbf{e}}_{s i, \perp}^{T}
\end{array}\right] \mathbf{p}_{g h} \quad \text { and } \quad \hat{\mathbf{e}}_{s i, \perp}=\left[\begin{array}{c}
\sin \hat{\theta}_{s i} \\
-\cos \hat{\theta}_{s i}
\end{array}\right] .}
\end{gathered}
$$

The coarse estimate of the tile's curvature $\tilde{\kappa}_{i}$ is obtained by averaging $\tilde{\kappa}_{l m n}$ over all the effective combinations of $l, m$ and $n$, i.e. $\forall l, m, n$, where $\left|v_{l m}\right|,\left|v_{m n}\right|$ and $\left|v_{l m}+v_{m n}\right| \gg 0$. The curvature estimated from a single tile is heavily distorted by noise. To get a stable estimate, an extra smoothing step is applied, exploiting the geometry equality

$$
\mathbf{p}_{s}^{(x y)}=\kappa_{i}^{-1} \mathbf{e}_{s i}+\mathbf{p}_{i}^{(x y)}=\frac{\sum_{\forall \mathcal{T}_{i}} \mathbf{e}_{s i}+\kappa_{i} \mathbf{p}_{i}^{(x y)}}{\sum_{\forall \mathcal{T}_{i}} \kappa_{i}} .
$$

The tile's curvature estimate can be refined as

$$
\hat{\kappa}_{i}=\left\|\left(\sum_{\forall \mathcal{T}_{j}} \tilde{\kappa}_{j}\right)^{-1}\left(\sum_{\forall \mathcal{T}_{j}} \hat{\mathbf{e}}_{s j}+\tilde{\kappa}_{j} \mathbf{p}_{j}^{(x y)}\right)-\mathbf{p}_{i}^{(x y)}\right\|^{-1} .
$$

Finally, the transmitter's position can be estimated by replacing $\theta_{s i}$ and $\kappa_{i}$ in (8) with their estimates $\hat{\theta}_{s i}$ and $\hat{\kappa}_{i}$.

\section{NUMERICAL RESULTS}

We simulate SWP with three different arrays orientated along the $x$-axis: a URA with aperture size in each dimension $A_{x}=1.5 \mathrm{~m}, A_{y}=0.3 \mathrm{~m}$ and two ULAs with aperture lengths $0.3 \mathrm{~m}$ and $1.5 \mathrm{~m}$. All arrays have $\lambda / 4$ antenna spacing. The tiles are constructed by $3 \times 3$ elements for the URA and $1 \times 3$ for the ULAs. A single antenna transmitter is deployed at distances from $1 \mathrm{~m}$ to $100 \mathrm{~m}$ and with DoAs from $0^{\circ}$ to $90^{\circ}$, transmitting a single-carrier signal with $10 \mathrm{dBm}$ transmit power at $1 \mathrm{GHz}$ carrier frequency. Free-space pathloss and a noise variance of $-123.2 \mathrm{dBm}$ are assumed. For each parameter set 100 Monte Carlo runs have been conducted.

First the performance of the URA is assessed. Fig. 2 shows the root mean square errors (RMSEs) of CoA positioning and a CoA initialized ML estimator, as well as the exact and approximated CRBs. With increasing $d_{s}$, the CoA positioning

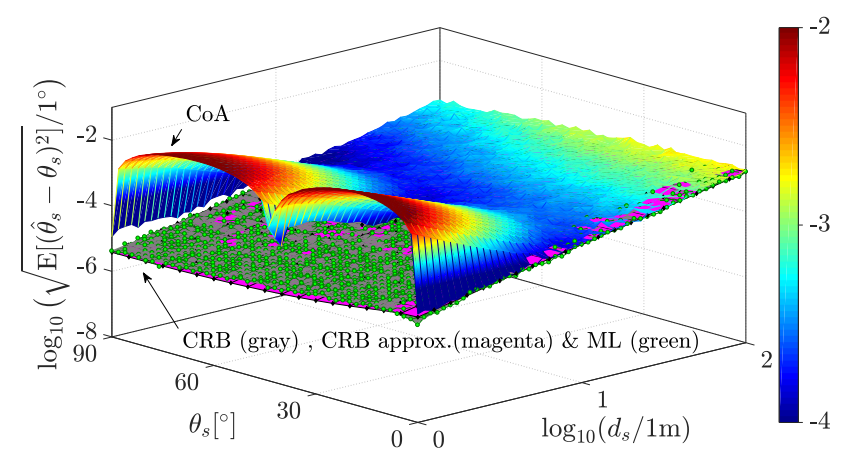

(a) DoA estimation error

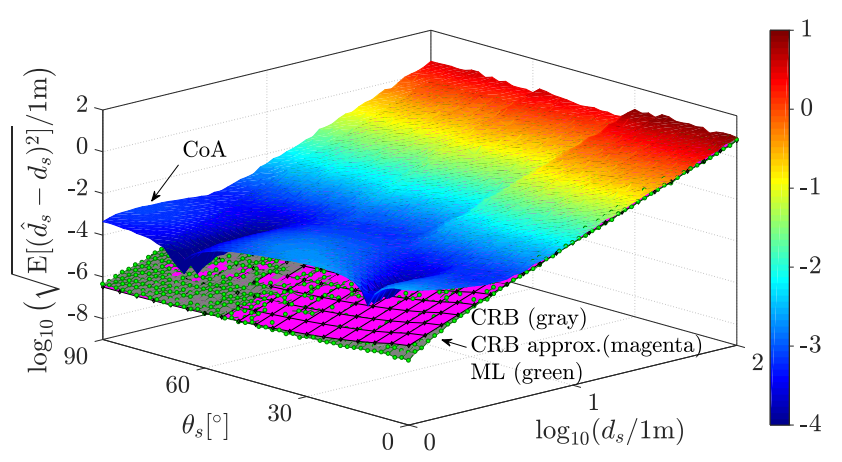

(b) Distance estimation error

Fig. 2: A URA along $x$-axis with $A_{x}=1.5 \mathrm{~m}, A_{y}=0.3 \mathrm{~m}$, $f_{c}=1 \mathrm{GHz}$ and spacing of $\lambda / 4$.

RMSEs first decrease due to a decreasing model error and then increase because of the reducing SNR and worsen geometry. For small $d_{s}$, the model error varies with $\theta_{s}$, but only leads to small estimation errors. By applying an ML estimator in addition, the RMSEs approach the CRBs. When both $d_{s}$ and $\theta_{s}$ are small, a slight Taylor approximation error from (10) is observed for the distance CRB. At most of the evaluation points, approximated and exact CRBs coincide, which verifies Corollary 1.

Next we compare the CoA positioning to traditional low complexity SWP algorithms with the ULAs, since most algorithms apply the Fresnel approximation on ULAs, [8], [10]. To remove the outliers occurring at small $\theta_{s}$, the RMSEs are calculated only for DoAs range between $30^{\circ}$ and $90^{\circ}$. Fig. 3 shows the performance of CoA positioning, the Fresnel based approach in [10], the CoA initialized ML estimator and the CRBs for different $d_{s}$. The Fresnel based approach estimates the DoA with the plane wave model, like the traditional farfield DoA estimation. The Fresnel based approach has a larger model error for larger arrays. In contrast, the CoA positioning only experiences model mismatch within individual tiles, independently of the total aperture. Therefore, the CoA positioning outperforms the Fresnel based approach for shorter distances and larger arrays. At larger distances, the model error is no longer dominant and all algorithms perform similarly along the CRBs. For the $0.3 \mathrm{~m}$ ULA at small $\theta_{s}$ and $d_{s}>50 \mathrm{~m}$, the ratio $d_{s} / \tilde{A}$ is so small, that none of the three algorithms is able to effectively estimate the distance. As a final result, for 


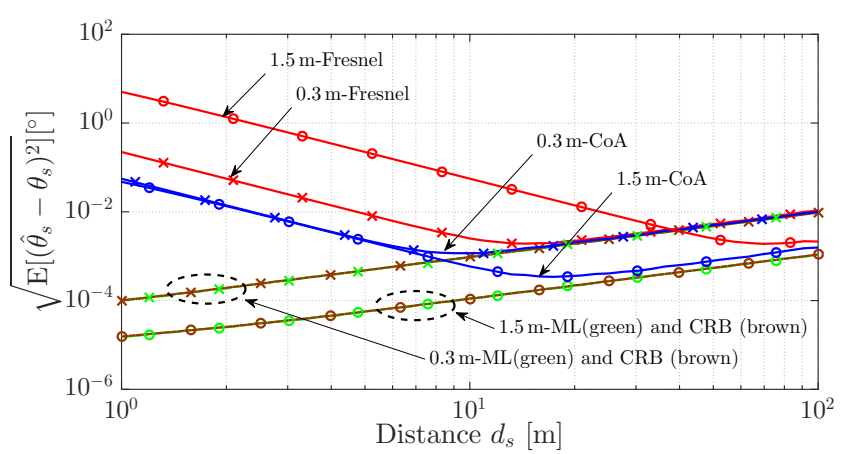

(a) DoA estimation error

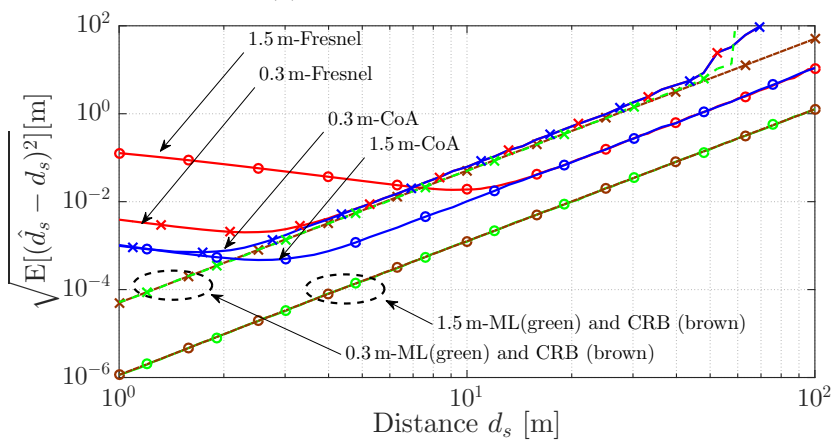

(b) Distance estimation error

Fig. 3: Two ULAs along $x$-axis with $A=0.3 \mathrm{~m}$ and $1.5 \mathrm{~m}$, $f_{c}=1 \mathrm{GHz}$ and spacing of $\lambda / 4$.

a $1.5 \mathrm{~m}$ sized array, the distance estimate can be achieved with a sub-meter RMSE by the CoA positioning up to $50 \mathrm{~m}$, which meets the accuracy expectation of $5 \mathrm{G}$ suggested in [2]. The CoA initialized ML estimator extends the applicable distance to $100 \mathrm{~m}$.

\section{CONCLUSION}

We proposed a CoA positioning algorithm, where the transmitter position is directly estimated from the wavefront curvature. Compared to conventional methods, CoA positioning does not require multiple anchors, synchronization, nor coordination between communication entities. Simplified CRBs show that for an arbitrary CSA, the achievable SWP performance only depends on the relative geometry and moments of the antennas' spatial distribution. Numerical results prove that the low complexity CoA positioning is effective for the considered applications and outperforms the Fresnel approximation based algorithms by overcoming the model mismatch. Hence, the proposed CoA positioning is suitable for realtime transmitter positioning in $5 \mathrm{G}$.

\section{APPENDIX}

We apply the second-order Taylor expansion to $d_{s l}$ at $d_{l}=0$

$$
d_{s l} \approx d_{s}-d_{l} \cos \left(\theta_{l}-\theta_{s}\right)+\frac{1}{2} \sin ^{2}\left(\theta_{l}-\theta_{s}\right) d_{l}^{2} / d_{s}
$$

and define $\quad a_{l} \triangleq 1-\frac{d_{l}^{2} \sin ^{2} \theta_{s}}{2 d_{s}^{2}} \quad$ and $\quad b_{l} \triangleq \frac{d_{l}^{2} \sin \theta_{s} \cos \theta_{s}}{d_{s}}$.
By exploiting the symmetry of SLAs, we can write

$$
\sum_{l=1}^{L} \frac{\partial d_{s l}}{\partial \mathbf{p}_{s}} \sum_{m=1}^{L} \frac{\partial d_{s m}}{\partial \mathbf{p}_{s}^{T}} \approx\left(\begin{array}{cc}
\left(\sum_{l=1}^{L} a_{l}\right)^{2} & \sum_{l=1}^{L} a_{l} \sum_{m=1}^{L} b_{m} \\
\sum_{l=1}^{L} a_{l} \sum_{m=1}^{L} b_{m} & \left(\sum_{l=1}^{L} b_{l}\right)^{2}
\end{array}\right)
$$$$
\text { and } \sum_{l=1}^{L} \frac{\partial d_{s l}}{\partial \mathbf{p}_{s}} \frac{\partial d_{s l}}{\partial \mathbf{p}_{s}^{T}} \approx \sum_{l=1}^{L}\left(\begin{array}{cc}
a_{l}^{2} & a_{l} b_{l} \\
a_{l} b_{l} & d_{l}^{2} \sin ^{2} \theta_{s}+b_{l}^{2}
\end{array}\right) \text {. }
$$

The CRB of $\mathbf{p}_{s}$ can be derived as

$$
\begin{aligned}
& \mathrm{CRB}\left[\mathbf{p}_{s}\right] \approx \frac{2 \sigma^{2} d_{s}^{2}}{S^{2} L \tilde{A}^{4}\left(M_{4}-M_{2}^{2}\right)} \\
& \times\left(\begin{array}{cc}
d_{s}^{-4} / 4 & \cot \theta_{s} d_{s}^{-3} / 2 \\
\cot \theta_{s} d_{s}^{-3} / 2 & \frac{\tilde{A}^{-2} M_{2}}{M_{4}-M_{2}^{2}}+\cot ^{2} \theta_{s} d_{s}^{-2}
\end{array}\right)^{-1} .
\end{aligned}
$$

The CRB of DoA in (5) can be directly obtained by taking the second diagonal entity of (11). The distance CRB is derived by taking the first diagonal entity of (11)

$$
\mathrm{CRB}\left[d_{s}\right] \approx \frac{8 \sigma^{2} d_{s}^{6}}{S^{2} L \tilde{A}^{4}\left(M_{4}-M_{2}^{2}\right)}\left(1+\frac{\left(M_{4}-M_{2}^{2}\right) \cot ^{2} \theta_{s}}{\left(d_{s} / \tilde{A}\right)^{2} M_{2}}\right) .
$$

Equation (6) is obtained from (12) with the assumption $d_{s} \gg$ $\tilde{A}$, which completes the proof.

\section{REFERENCES}

[1] F. Boccardi, R. W. Heath, A. Lozano, T. L. Marzetta, and P. Popovski, "Five disruptive technology directions for 5G," IEEE Commun. Mag., vol. 52, no. 2, pp. 74-80, February 2014.

[2] J. del Peral-Rosado, R. Raulefs, J. Lpez-Salcedo, and G. Seco-Granados, "Survey of cellular mobile radio localization methods: from $1 \mathrm{G}$ to $5 \mathrm{G}$," IEEE Commun. Surveys Tuts., vol. 20, no. 2, pp. 1124-1148, 2018.

[3] M. Win et al., "Network localization and navigation via cooperation," IEEE Commun. Mag., vol. 49, no. 5, pp. 56-62, May 2011.

[4] C. Gentner et al., "Multipath assisted positioning with simultaneous localization and mapping," IEEE Trans. Wireless Commun., vol. 15, no. 9, pp. 6104-6117, Sep. 2016.

[5] A. Shahmansoori et al., "Position and orientation estimation through millimeter-wave MIMO in 5G systems," IEEE Trans. Wireless Commun., vol. 17, no. 3, pp. 1822-1835, March 2018.

[6] Y. Han, Y. Shen, X. Zhang, M. Z. Win, and H. Meng, "Performance limits and geometric properties of array localization," IEEE Trans. Inf. Theory, vol. 62, no. 2, pp. 1054-1075, Feb 2016.

[7] X. Yin et al., "Scatterer localization using large-scale antenna arrays based on a spherical wave-front parametric model," IEEE Trans. Wireless Commun., vol. 16, no. 10, pp. 6543-6556, Oct 2017.

[8] E. Grosicki, K. Abed-Meraim, and Y. Hua, "A weighted linear prediction method for near-field source localization," IEEE Trans. Signal Process., vol. 53, no. 10, pp. 3651-3660, Oct 2005.

[9] J. Chen, X. Zhu, and X. Zhang, "A new algorithm for joint rangeDoA-frequency estimation of near-field sources," EURASIP Journal on Advances in Signal Processing, vol. 2004, no. 3, p. 105173, Mar 2004.

[10] K. Deng, Q. Yin, and H. Wang, "Closed form parameters estimation for near field sources," in 2007 IEEE International Symposium on Circuits and Systems, May 2007, pp. 3251-3254.

[11] Y. Hsu, K. T. Wong, and L. Yeh, "Mismatch of near-field bearing-range spatial geometry in source-localization by a uniform linear array," IEEE Trans. Antennas Propag., vol. 59, no. 10, pp. 3658-3667, Oct 2011.

[12] P. R. Singh, Y. Wang, and P. Charg, "Performance enhancement of approximated model based near-field sources localisation techniques," IET Signal Processing, vol. 11, no. 7, pp. 825-829, 2017.

[13] J.-P. Delmas, M. N. El Korso, H. Gazzah, and M. Castella, "CRB analysis of planar antenna arrays for optimizing near-field source localization," Signal Processing, vol. 127, pp. 117 - 134, Oct. 2016.

[14] T. E. Tuncer and B. Friedlander, Classical and Modern Direction-ofArrival Estimation. Academic Press, 2009. 